de Groot M, Wessel J. Genetic Testing and Type 2 Diabetes Risk Awareness. The Diabetes Educator. 2014 Mar 19. Available from: http:// dx.doi.org/10.1177/0145721714527643

\title{
Genetic Testing and Type 2 Diabetes Risk Awareness
}

\section{Purpose}

The purpose of this study was to examine the motivational, attitudinal, and behavioral predictors of interest in genetic testing (GT) in those with and without awareness of their risk for type 2 diabetes (T2DM).

\section{Methods}

A convenience sample of adults visiting emergency departments, libraries, or an online research registry was surveyed. Responses from adults without diabetes who reported 1 or more risk factors for T2DM (eg, family history, body mass index $>25$ ) were included in the analyses $(n=265)$.

\section{Results}

Participants were $37 \pm 11$ years old, white (54\%), and female (69\%), with some college education (53\%) and an annual income below $\$ 25000$ (44\%). Approximately half $(52 \%)$ expressed interest in GT for T2DM. Individuals were stratified by perceived risk for T2DM (risk aware or risk unaware). Among the risk aware, younger age $(P<.04)$ predicted greater interest in GT. Among the risk unaware, family history of T2DM $(P<$ $.008)$ and preference to know genetic risk $(P<.0002)$ predicted interest in GT. Both groups identified the need for low-cost GT.

\section{Conclusions}

GT is an increasingly available and accurate tool to predict T2DM risk for patients. In this sample, GT was a
Mary de Groot, PhD

Jennifer Wessel, PhD, MPH

From the School of Medicine, Indiana University, Indianapolis, IN, USA (Dr de Groot, Dr Wessel), and the Fairbanks School of Public Health, Indiana University, Indianapolis, IN, USA (Dr Wessel).

Correspondence to Jennifer Wessel, PhD, MPH, Fairbanks School of Public Health, Indiana University Purdue University at Indianapolis, 714 North Senate Avenue, Suite EF250, Indianapolis, IN 46202, USA (wesselj@iu.edu).

Acknowledgments: The study was funded by the Center for Urban Health and supported by the Indiana University-Purdue University Indianapolis Signature Center Initiative, the Indiana Clinical Translational Sciences Institute, and the National Institute of Diabetes and Digestive and Kidney Diseases (R18DK092765). The survey instrument was designed with the Research Electronic Data Capture professional survey instrument, hosted by the Indiana Clinical Translational Sciences Institute HUB. We are grateful to Dr James Jones, vice chair of the emergency department at Wishard Hospital, and the management at Indianapolis Public Libraries for permitting us to recruit participants in their organizations.

DOI: $10.1177 / 0145721714527643$

(C) 2014 The Author(s) 
salient tool for those with and without awareness of their T2DM risk. Financial accessibility is critical to use of this tool for both groups.

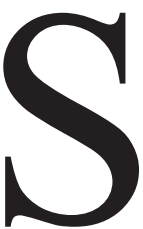

ixty-eight percent of Americans are overweight or obese, with approximately 26 million estimated to have diagnosed or undiagnosed type 2 diabetes (T2DM). An additional estimated 79 million adults older than 30 years have prediabetes, placing them at significant risk of developing T2DM. By 2050, an estimated 1 in 3 people will be diagnosed with T2DM. ${ }^{1}$ In 2012 alone, T2DM cost $\$ 245$ billion, accounting for 1 in 5 health care dollars. $^{2}$ At the same time, there is strong evidence that behavioral interventions can effectively delay or prevent the onset of T2DM. ${ }^{3}$ Individuals with prediabetes represent the next wave of patients to be diagnosed with T2DM. Those with low perceived risk of T2DM are the least likely to engage in diabetes prevention strategies or formal programs and therefore represent the highest likelihood of progressing to diagnosis of T2DM.

Genetic testing represents a rapidly growing and increasingly accessible form of information about T2DM risk. Through the recent successes of genomewide association studies, approximately 60 single-nucleotide polymorphisms have been robustly associated with T2DM $\mathrm{DM}^{4-16}$ and related traits. ${ }^{17-21}$ More single-nucleotide polymorphisms will be identified in the coming years. Genetic factors alone can significantly predict incident T2DM over the life course $\mathrm{e}^{22-33}$ and are independent of family history. ${ }^{34}$ The recent advances in genetic technology have decreased costs of genotyping and whole genome sequencing exponentially, ${ }^{35}$ where the expected cost will be approximately $\$ 1000$ in the next few years. ${ }^{36-38}$ Lowered costs and recent discoveries are already leading to increasingly cost-effective ${ }^{39,40}$ clinical applications. Additionally, there is an increasing demand to utilize genomics by providers and patients. ${ }^{41}$

Knowledge of personal genetic risk, coupled with the means to behaviorally reduce risk (ie, the availability of diabetes prevention programs), has the potential to synergistically motivate individuals to engage in diabetes prevention. To date, few studies have examined the utility of genetic testing as a tool to assist individuals in precisely estimating risk of disease onset and motivating behavior change to prevent the onset of disease. ${ }^{42}$ Only 1 study has evaluated risk perception and T2DM prevention. ${ }^{43}$ Grant and colleagues classified 108 adults with high or low genetic risk and then compared their motivation to engage in lifestyle changes, as well as their participation in a diabetes prevention program, to controls. ${ }^{43}$ High-risk participants showed significant increases in motivation to make lifestyle changes and participate in the diabetes prevention program $(P=.01)$ compared to controls, but no significant differences in attendance were observed. The genetic counseling session was positively received, and it improved perceptions of control over the onset of T2DM in the high-risk group. ${ }^{44}$ This suggests that genetic risk information may contribute to motivating behavior change. However, the sample predominantly comprised people who already perceived themselves to be at high risk for T2DM, and it sheds little light on those who underestimate their risk of T2DM despite the presence of absolute risk factors. Those who underestimate their risk pose the greatest challenge to health care systems and providers, as they are the least likely to engage in preventive behaviors, potentially resulting in greater costs and poorer health outcomes.

Taken together, the epidemic of obesity and metabolic disorder that precede the development of T2DM illuminates the need to assist high-risk individuals to identify their risk for the development of T2DM and engage in diabetes prevention. Genetic testing is emerging as a cost-effective tool to assist individuals in precisely estimating this risk in addition to known behavioral and environmental factors. To date, little is known about motivation, attitudes, and behaviors related to genetic testing among those with T2DM risk factors. The purpose of the current study was to examine the motivational, attitudinal, and behavioral predictors of interest in genetic testing among those with one or more risk factors for T2DM, stratified by perceived risk for T2DM: risk aware (RA) or risk unaware (RUA).

\section{Methods}

\section{Study Sample}

The total study sample was 649 adults who were aged 18 to 65 years and residents of Indiana. A convenience sample of participants from 14 public libraries $(n=342$, $53 \%)$ and the emergency room of Wishard Hospital ( $\mathrm{n}=$ $62,10 \%)$ and individuals responding online $(\mathrm{n}=245$, $37 \%$ ) through a research registry (INResearch.org) were recruited from June to July 2012. These 3 settings were 
chosen to maximize representation of diverse groups that have been underrepresented in prior genetic utilization research. INResearch is a registry of more than 900 research volunteers. The Indianapolis Public Library system has 23 sites around the city, offering resources and services for learning as well as to promote the social and economic interests of its communities. Wishard Hospital is the safety-net health system of Indianapolis, with a special emphasis on vulnerable populations. The Wishard Hospital emergency room and library sites in lower socioeconomic neighborhoods were specifically chosen to obtain the broadest representation of demographic, socioeconomic status, and literacy. The study was approved by the Indiana University Institutional Review Board.

\section{Questionnaire}

Consistent with standard survey development procedures ${ }^{45}$ survey questions were developed by one of the authors, using extant literature on the assessment of attitudes, beliefs, and behaviors associated with genetic testing in T2DM and other chronic diseases. An initial sample of 75 items were created and distributed to 5 experts in the fields of psychology, sociology, and anthropology for evaluation. Following revisions, items were piloted in a group of 10 participants for clarity of wording. Based on feedback, items were then edited, resulting in a total of 59 items. Demographic, personal, and family medical history and psychosocial and behavioral factors related to interest in genetic testing for T2DM risk prediction were included in the final survey instrument. Demographic, perceived health, diabetes risk, and medical history questions were adapted from standardized questionnaires. Genetics knowledge was adapted from existing questionnaires.

To capture the widest variety of attitudinal and behavioral constructs related to genetic testing, psychosocial questions were informed Health Belief Model, the Theory of Planned Behavior and Reasoned Action, the Social Cognitive Theory, the Transactional Model of Stress and Coping, and the Precaution Adoption Process Model. Questions relating to constructs developed or adopted by these behavior change models included perceived health, perceived risk, awareness, knowledge, worry, motivation, utility, barriers, trust, and intention. The questions were designed to query participants about genetics, genetic testing, T2DM, and T2DM risk.

Questions relating to worry about risk of T2DM were measured with a 7-point Likert scale. All other questions used a 5-point Likert scale. Items $(n=5)$ assessing knowledge of genetics or genetic literacy were scored with 1 point given to each question answered correctly and summed to create a genetics knowledge total score $($ range $=0-5)$. A score of 4 or greater indicated high levels of knowledge about genetic testing.

Questionnaires were self-administered through paper or web-based versions, available in English or Spanish (1.8\% of total surveys). The online questionnaire was distributed to prospective participants registered at Indiana's volunteer research participant registry. The printed version of the questionnaire was handed to eligible participants by trained interviewers at the public libraries or the emergency department waiting room of Wishard Hospital. After completing the survey, each study participant was compensated with a \$10 gift card and offered educational materials describing diabetes, risk factors and symptoms, and the Genetic Information Nondiscrimination Act (or GINA bill). All materials were available in both English and Spanish.

\section{Statistical Analyses}

SAS 9.3 was used for all statistical analyses. A total sample of 649 individuals completed the questionnaire. Individuals were excluded based on self-reported diabetes $(\mathrm{n}=50)$ and body mass index $<15 \mathrm{~kg} / \mathrm{m}^{2}(\mathrm{n}=1)$. Of these, only individuals self-reporting the following characteristics were included in the current analyses: selfreported race as black or white, 1 or more risk factors for T2DM (body mass index, family history of T2DM, or gestational diabetes), and a response to the item evaluating perceived risk of developing T2DM as likely (RA) or unlikely (RUA). This resulted in a final sample of 265 participants. RUA individuals were defined as those reporting low perceived risk and having at least 1 risk factor T2DM (body mass index $>25 \mathrm{~kg} / \mathrm{m}^{2}$, family history, or gestational diabetes; $\mathrm{n}=169$ ). RA individuals were defined as rating themselves with high perceived risk and at least 1 risk factor for T2DM $(n=69)$. The dependent variable was individuals reporting interest in genetic testing to predict future risk of developing T2DM (yes vs no/not sure). Univariate logistic regression analyses were performed by RA/RUA status. Independent variables $(n=47)$ included demographic variables, motivation, knowledge, or intention to engage in genetic testing for T2DM risk prediction. Variables reaching significance at $P=.001$ or better (Bonferroni correction for 46 independent tests) or were psychosocially relevant and age, sex, ethnicity, recruitment location were entered 
Table 1

Characteristics of the Study Population, No. (\%)

\begin{tabular}{|c|c|c|c|}
\hline & Risk Unaware $(n=169)$ & Risk Aware $(n=96)$ & $\boldsymbol{P}$ \\
\hline $\mathrm{Age}^{\mathrm{a}} \mathrm{y}$ & $36.1 \pm 10.9$ & $37.2 \pm 10.5$ & .39 \\
\hline Sex, female & $117(69.2)$ & 77 (80.2) & .05 \\
\hline \multicolumn{4}{|l|}{ Income } \\
\hline$<\$ 25000$ & $66(39.1)$ & $44(45.8)$ & \multirow[t]{5}{*}{.36} \\
\hline$\$ 25000-\$ 50000$ & $47(27.8)$ & 27 (28.1) & \\
\hline$\$ 50001-\$ 75000$ & 19 (11.2) & $6(6.3)$ & \\
\hline$\$ 75001-\$ 100000$ & $16(9.5)$ & 12 (12.5) & \\
\hline$>\$ 100,000$ & $21(12.4)$ & $7(7.3)$ & \\
\hline \multicolumn{4}{|l|}{ Health insurance } \\
\hline Commercial & $96(57.1)$ & $49(51.0)$ & \multirow[t]{4}{*}{.52} \\
\hline Medicaid & 26 (15.5) & 21 (21.9) & \\
\hline Medicare & $10(6.0)$ & $4(4.2)$ & \\
\hline Other & $36(21.4)$ & $22(22.9)$ & \\
\hline \multicolumn{4}{|l|}{ Ethnicity } \\
\hline Black & $80(47.3)$ & $30(31.3)$ & \multirow[t]{2}{*}{$.01^{*}$} \\
\hline White & $89(52.7)$ & $66(68.8)$ & \\
\hline \multicolumn{4}{|l|}{ Education } \\
\hline College or more & $85(50.3)$ & $46(47.9)$ & \multirow[t]{4}{*}{.96} \\
\hline Some college & $39(23.1)$ & $22(22.9)$ & \\
\hline Completed high school & 29 (17.2) & 17 (17.7) & \\
\hline Some/less high school & $16(9.5)$ & 11 (11.5) & \\
\hline \multicolumn{4}{|l|}{ Marital status } \\
\hline Cohabitating & $13(7.7)$ & $10(10.4)$ & \multirow[t]{3}{*}{.75} \\
\hline Married & $59(34.9)$ & $32(33.3)$ & \\
\hline Single & $97(57.4)$ & $54(56.3)$ & \\
\hline
\end{tabular}

into multivariate logistic regression models for RA and RUA separately.

\section{Results}

For the 265 participants in these analyses, $52 \%$ had a family history of T2DM; $83 \%$ had a body mass index $>25$ $\mathrm{kg} / \mathrm{m}^{2} ; 3 \%$ reported a history of gestational diabetes; and $58 \%$ reported low perceived risk for developing T2DM. Demographic characteristics of the sample by risk estimation group are shown in Table 1, where $64 \%$ are RUA and $36 \%$ are RA. A greater proportion of those with RA were white $(P<.01)$. A larger proportion of those with RUA were male $(P=.05)$. Those with RA were more likely to indicate interest in genetic testing (58\%) compared to those with RUA ( $45 \%, P<.04$, data not shown).
Multivariate analyses were conducted to identify predictors of interest in genetic testing for RA and RUA individuals in separate models in which demographic characteristics and significant predictors from the univariate models were entered simultaneously. For RA individuals (see Table 2), interest in genetic testing was best predicted by younger age $(P=.04)$ and no cost of genetic testing to the individual $(P=.0001)$. Sex, ethnicity, or increased motivation to engage in genetic testing due to accuracy of the test was not associated with interest among the RA individuals. Among those with RUA (see Table 3), predictors of interest in genetic testing included increased motivation by positive family history of T2DM $(P=.008)$, preference to know risk before diagnosis $(P=.0002)$, and no cost of genetic testing to the individual $(P=.0002)$. Age, sex, ethnicity, recruitment location, familiarity with 
Table 2

Multivariate Analyses of Demographic and Psychosocial Associations With Interest in Genetic Testing for Type 2 Diabetes Risk Prediction Among Risk Aware ${ }^{\mathrm{a}}(\mathrm{n}=96)$

\begin{tabular}{|lcc|}
\hline & Odds Ratio (95\% Confidence Interval) & P \\
\hline Age (per year) & $0.95(0.90,1.00)$ & $.0452^{*}$ \\
Female vs male & $0.99(0.25,3.90)$ & .9926 \\
Black vs white & $0.31(0.10,0.97)$ & .1939 \\
Recruitment location & $2.53(0.60,10.6)$ & .2052 \\
Accuracy of test increases motivation for genetic testing & $2.48(0.79,7.82)$ & .1215 \\
Would be interested in genetic testing if free of cost & $34.2(5.5,210.7)$ & $.0001^{*}$ \\
\hline${ }^{*}$ Adjusted for age, sex, ethnicity, and recruitment location. & & \\
${ }^{*} P<.05$. & & \\
\hline
\end{tabular}

Table 3

Multivariate Analyses of Demographic and Psychosocial Associations With Interest in Genetic Testing for Type 2 Diabetes Risk Prediction Among Risk Unaware ${ }^{\mathrm{a}}(\mathrm{n}=169)$

\begin{tabular}{|lcc|}
\hline & Odds Ratio (95\% Confidence Interval) & $\boldsymbol{P}$ \\
\hline Age & $1.01(0.97,1.06)$ & .5122 \\
Sex & $1.75(0.65,4.74)$ & .2701 \\
Black vs white & $0.76(0.30,1.93)$ & .5615 \\
Recruitment location & $0.85(0.32,2.27)$ & .7420 \\
Currently motivated to have genetic testing for type 2 diabetes risk due to ... & $3.95(1.43,10.9)$ & .0079 \\
$\quad$ Family history of type 2 diabetes & $5.58(2.23,13.9)$ & $.0002^{*}$ \\
$\quad$ Prefer to know risk before diagnosis & $0.76(0.28,2.04)$ & .5869 \\
$\quad$ Know someone with diabetes complications & $24.9(4.46,128.0)$ & $.0002^{*}$ \\
Would be interested in genetic testing if ... & $2.67(0.49,14.5)$ & .2537 \\
$\quad$ Free of cost & & \\
$\quad$ Diabetes is preventable & & \\
\hline${ }^{2}$ Adjusted for age, sex, ethnicity, and recruitment location. & & \\
${ }^{*} P<.05$. & & \\
\hline
\end{tabular}

someone with diabetes complications, and belief that diabetes is preventable were not significantly associated with genetic testing interest among the RUA group.

\section{Discussion}

In the current study, demographic and psychosocial predictors of genetic testing utilization were examined in those with T2DM risk unawareness compared to those who correctly identified themselves as having elevated risk. Differences in the patterns of interest in genetic testing were observed across these 2 groups suggesting that use of genetic testing is salient for both groups but the usefulness differs by patients' perceptions of their risk for T2DM. For example, data indicated that among those with RA, younger participants were more receptive to genetic testing, suggesting that older adults may need additional information about the utility and relevance of genetic testing. This is noteworthy in light of the additional risk factors for T2DM with increasing age. 
In contrast, those with RUA were more likely to be interested in genetic testing if they had a family history of T2DM and some interest in knowing their risk. Although these individuals did not perceive themselves to be at risk for T2DM, their awareness of traditional risk factors (eg, family history) in conjunction with curiosity about their own status increased their likelihood to make use of genetic testing as an additional source of information about their health. For this crucial group in which risk factors are high and prevention steps could be taken to significantly reduce risk and prevent disease onset, these findings suggest that primary care providers may use traditional risk factor information in conjunction with accessible genetic testing to demonstrate to patients the relevance that diabetes prevention programs may have on preserving health and reducing risk for T2DM. In this group, genetic testing has the potential to engage the patients' curiosity about their predisposition about disease and, in turn, enhance their motivation to engage in diabetes prevention.

Another finding from these data is the need for financially accessible genetic testing. Both groups (RA and RUA) indicated greater interest in genetic testing if cost to the individual was minimal. As technological innovations lower the cost of testing, health care providers, laboratories, and insurers will need to attend to the costbenefit ratio of supplementing the cost of genetic testing to enhance the accessibility and desirability of testing for T2DM genetic risk.

There are 2 primary limitations to the current study. Data were collected from a convenience sample of research volunteers and those attending an emergency department or public library. While this may introduce bias, data were intentionally collected across the spectrum of sociodemographic characteristics, and, indeed, differences in demographic characteristics by recruitment location were observed. This variable was entered into multivariate analyses, and it was not a significant predictor of genetic testing in either risk group. Second, all data, including body mass index and history of T2DM, were self-reported and not measured independently by the research staff. Body mass index was estimated from self-reported height and weight and should be interpreted as approximations to actual values.

\section{Implications for Diabetes Education}

As rates of obesity and prediabetes continue to rise, there is a need to fully utilize all available tools to identify risk of T2DM and mitigate that risk through diabetes prevention. Those who are RUA are the least likely to engage in diabetes prevention. Genetic testing is a new tool that will become increasingly available to health care providers and patients, and it represents greater accuracy of prediction than that of conventional risk factors. The current study found that RA and RUA groups both identified genetic testing as a salient tool for the prediction of T2DM, although predictors differed by group. Future use of genetic testing to estimate risk of T2DM will depend on the perceived utility and financial accessibility of this tool by patients. Genetic testing has the potential to add to the set of metrics that health care providers can use to empower patients with advanced knowledge about their health, to identify risk, and to prevent T2DM.

\section{References}

1. National Diabetes Information Clearinghouse. National diabetes statistics, 2011. http://diabetes.niddk.nih.gov/dm/pubs/statistics/. Accessed December 28, 2013.

2. American Diabetes Association. Economic costs of diabetes in the US in 2012. Diabetes Care. 2013;36:1033-1046.

3. Knowler WC, Barrett-Connor E, Fowler SE, et al. Reduction in the incidence of type 2 diabetes with lifestyle intervention or metformin. N Engl J Med. 2002;346:393-403.

4. Scott RA, Lagou V, Welch RP, et al. Large-scale association analyses identify new loci influencing glycemic traits and provide insight into the underlying biological pathways. Nat Genet. 2012;44:991-1005.

5. Morris AP, Voight BF, Teslovich TM, et al. Large-scale association analysis provides insights into the genetic architecture and pathophysiology of type 2 diabetes. Nat Genet. 2012;44:981-990.

6. Voight BF, Scott LJ, Steinthorsdottir V, et al. Twelve type 2 diabetes susceptibility loci identified through large-scale association analysis. Nat Genet. 2010;42:579-589.

7. Zeggini E, Scott LJ, Saxena R, et al. Meta-analysis of genomewide association data and large-scale replication identifies additional susceptibility loci for type 2 diabetes. Nat Genet. 2008;40:638-645.

8. Saxena R, Voight BF, Lyssenko V, et al. Genome-wide association analysis identifies loci for type 2 diabetes and triglyceride levels. Science. 2007;316:1331-1336.

9. Scott LJ, Mohlke KL, Bonnycastle LL, et al. A genome-wide association study of type 2 diabetes in Finns detects multiple susceptibility variants. Science. 2007;316:1341-1345.

10. Zeggini E, Weedon MN, Lindgren CM, et al. Replication of genome-wide association signals in UK samples reveals risk loci for type 2 diabetes. Science. 2007;316:1336-1341.

11. Sladek R, Rocheleau G, Rung J, et al. A genome-wide association study identifies novel risk loci for type 2 diabetes. Nature. 2007;445:881-885.

12. Gloyn AL, Weedon MN, Owen KR, et al. Large-scale association studies of variants in genes encoding the pancreatic beta-cell KATP channel subunits Kir6.2 (KCNJ11) and SUR1 (ABCC8) confirm that the $K C N J 11 \mathrm{E} 23 \mathrm{~K}$ variant is associated with type 2 diabetes. Diabetes. 2003;52:568-572. 
13. Altshuler D, Hirschhorn JN, Klannemark M, et al. The common PPARgamma Pro12Ala polymorphism is associated with decreased risk of type 2 diabetes. Nat Genet. 2000;26:76-80.

14. Grant SF, Thorleifsson G, Reynisdottir I, et al. Variant of transcription factor 7-like 2 (TCF7L2) gene confers risk of type 2 diabetes. Nat Genet. 2006;38:320-323.

15. Helgason A, Palsson S, Thorleifsson G, et al. Refining the impact of TCF7L2 gene variants on type 2 diabetes and adaptive evolution. Nat Genet. 2007;39:218-225.

16. Reynisdottir I, Thorleifsson G, Benediktsson R, et al. Localization of a susceptibility gene for type 2 diabetes to chromosome 5q34q35.2. Am J Hum Genet. 2003;73:323-335.

17. Bouatia-Naji N, Rocheleau G, Van Lommel L, et al. A polymorphism within the G6PC2 gene is associated with fasting plasma glucose levels. Science. 2008;320:1085-1088.

18. Prokopenko I, Langenberg C, Florez JC, et al. Variants in MTNR1B influence fasting glucose levels. Nat Genet. 2009;41:7781.

19. Dupuis J, Langenberg C, Prokopenko I, et al. New genetic loci implicated in fasting glucose homeostasis and their impact on type 2 diabetes risk. Nat Genet. 2010;42:105-116.

20. Manning AK, Hivert MF, Scott RA, et al. A genome-wide approach accounting for body mass index identifies genetic variants influencing fasting glycemic traits and insulin resistance. Nat Genet. 2012;44:659-69.

21. Bouatia-Naji N, Bonnefond A, Cavalcanti-Proenca C, et al. A variant near MTNR1B is associated with increased fasting plasma glucose levels and type 2 diabetes risk. Nat Genet. 2009;41:89-94.

22. Lyssenko V, Jonsson A, Almgren P, et al. Clinical risk factors, DNA variants, and the development of type 2 diabetes. $N$ Engl J Med. 2008;359:2220-2232.

23. Vassy JL, Dasmahapatra P, Meigs JB, et al. Genotype prediction of adult type 2 diabetes from adolescence in a multiracial population. Pediatrics. 2012;130:e1235-e1242.

24. Vassy JL, Durant NH, Kabagambe EK, et al. A genotype risk score predicts type 2 diabetes from young adulthood: the CARDIA study. Diabetologia. 2012;55:2604-2612.

25. de Miguel-Yanes JM, Shrader P, Pencina MJ, et al. Genetic risk reclassification for type 2 diabetes by age below or above 50 years using 40 type 2 diabetes risk single nucleotide polymorphisms. Diabetes Care. 2011;34:121-125.

26. van Hoek M, Dehghan A, Witteman JC, et al. Predicting type 2 diabetes based on polymorphisms from genome-wide association studies: a population-based study. Diabetes. 2008;57:3122-3128.

27. Talmud PJ, Hingorani AD, Cooper JA, et al. Utility of genetic and non-genetic risk factors in prediction of type 2 diabetes: Whitehall II prospective cohort study. BMJ. 2010;340:b4838.
28. Vaxillaire M, Veslot J, Dina C, et al. Impact of common type 2 diabetes risk polymorphisms in the DESIR prospective study. Diabetes. 2008;57:244-254.

29. Meigs JB, Shrader P, Sullivan LM, et al. Genotype score in addition to common risk factors for prediction of type 2 diabetes. $N$ Engl J Med. 2008;359:2208-2219.

30. Pearson H. Genetic testing for everyone. Nature. 2008;453:570571.

31. Katsanis SH, Javitt G, Hudson K. Public health: a case study of personalized medicine. Science. 2008;320:53-54.

32. Positively disruptive [editorial]. Nat Genet. 2008;40:119.

33. Bonetta L. Getting up close and personal with your genome. Cell. 2008;133:753-756.

34. Belsky DW, Moffitt TE, Houts R, et al. Polygenic risk, rapid childhood growth, and the development of obesity: evidence from a 4-decade longitudinal study. Arch Pediatr Adolesc Med. 2012;166:515-521.

35. MacArthur DG, Lek M. The uncertain road towards genomic medicine. Trends Genet. 2012;28:303-305.

36. Kedes L, Liu ET. The Archon Genomics X PRIZE for whole human genome sequencing. Nat Genet. 2010;42:917-918.

37. Kedes L, Liu E, Jongeneel CV, Sutton G. Judging the Archon Genomics X PRIZE for whole human genome sequencing. Nat Genet. 2011;43:175.

38. Kedes L, Campany G. The new date, new format, new goals and new sponsor of the Archon Genomics X PRIZE competition. Nat Genet. 2011;43:1055-1058.

39. Brunham LR, Hayden MR. Medicine: whole-genome sequencing. The new standard of care? Science. 2012;336:1112-1113.

40. Greeley SA, John PM, Winn AN, et al. The cost-effectiveness of personalized genetic medicine: the case of genetic testing in neonatal diabetes. Diabetes Care. 2011;34:622-627.

41. Grant R, Hivert M, Pandiscio J, Florez J, Nathan D, Meigs J. The clinical application of genetic testing in type 2 diabetes: a patient and physician survey. Diabetologia. 2009;52:2299-2305.

42. Scheuner MT, Sieverding P, Shekelle PG. Delivery of genomic medicine for common chronic adult diseases: a systematic review. JAMA. 2008;299:1320-1334.

43. Grant RW, O'Brien KE, Waxler JL, et al. Personalized genetic risk counseling to motivate diabetes prevention: a randomized trial. Diabetes Care. 2013;36:13-19.

44. Waxler JL, O'Brien KE, Delahanty LM, et al. Genetic counseling as a tool for type 2 diabetes prevention: a genetic counseling framework for common polygenetic disorders. J Genet Couns. 2012;21:684-691.

45. De Vellis RF. Scale Development: Theory and Applications. 3rd ed. Los Angeles, CA: Sage; 2012. 